\title{
New limits for neutrinoless tau decays
}

D. W. Bliss, G. Masek, H. P. Paar, S. Prell, and V. Sharma

University of California, San Diego, La Jolla, California 92093

D. M. Asner, J. Gronberg, T. S. Hill, D. J. Lange, R. J. Morrison, H. N. Nelson, T. K. Nelson, D. Roberts, and A. Ryd University of California, Santa Barbara, California 93106

R. Balest, B. H. Behrens, W. T. Ford, H. Park, J. Roy, and J. G. Smith

University of Colorado, Boulder, Colorado 80309-0390

J. P. Alexander, R. Baker, C. Bebek, B. E. Berger, K. Berkelman, K. Bloom, V. Boisvert, D. G. Cassel, D. S. Crowcroft, M. Dickson, S. von Dombrowski, P. S. Drell, K. M. Ecklund, R. Ehrlich, A. D. Foland, P. Gaidarev, R. S. Galik, L. Gibbons, B. Gittelman, S. W. Gray, D. L. Hartill, B. K. Heltsley, P. I. Hopman, J. Kandaswamy, P. C. Kim, D. L. Kreinick, T. Lee, Y. Liu, N. B. Mistry, C. R. Ng, E. Nordberg, M. Ogg, ' J. R. Patterson, D. Peterson, D. Riley, A. Soffer, B. Valant-Spaight, and C. Ward Cornell University, Ithaca, New York 14853

M. Athanas, P. Avery, C. D. Jones, M. Lohner, S. Patton, C. Prescott, J. Yelton, and J. Zheng University of Florida, Gainesville, Florida 32611

G. Brandenburg, R. A. Briere, A. Ershov, Y. S. Gao, D. Y.-J. Kim, R. Wilson, and H. Yamamoto Harvard University, Cambridge, Massachusetts 02138

T. E. Browder, Y. Li, and J. L. Rodriguez

University of Hawaii at Manoa, Honolulu, Hawaii 96822

T. Bergfeld, B. I. Eisenstein, J. Ernst, G. E. Gladding, G. D. Gollin, R. M. Hans, E. Johnson, I. Karliner, M. A. Marsh, M. Palmer, M. Selen, and J. J. Thaler University of Illinois, Urbana-Champaign, Illinois 61801

K. W. Edwards

Carleton University, Ottawa, Ontario, Canada K1S 5B6 and the Institute of Particle Physics, Canada

A. Bellerive, R. Janicek, D. B. MacFarlane, and P. M. Patel McGill University, Montréal, Québec, Canada H3A $2 T 8$ and the Institute of Particle Physics, Canada

\author{
A. J. Sadoff \\ Ithaca College, Ithaca, New York 14850
}

R. Ammar, P. Baringer, A. Bean, D. Besson, D. Coppage, C. Darling, R. Davis, S. Kotov, I. Kravchenko, N. Kwak, and L. Zhou University of Kansas, Lawrence, Kansas 66045

S. Anderson, Y. Kubota, S. J. Lee, J. J. O’Neill, R. Poling, T. Riehle, and A. Smith University of Minnesota, Minneapolis, Minnesota 55455

M. S. Alam, S. B. Athar, Z. Ling, A. H. Mahmood, S. Timm, and F. Wappler State University of New York at Albany, Albany, New York 12222

A. Anastassov, J. E. Duboscq, D. Fujino, ${ }^{\dagger}$ K. K. Gan, T. Hart, K. Honscheid, H. Kagan, R. Kass, J. Lee, M. B. Spencer, M. Sung, A. Undrus, ${ }^{\ddagger}$ R. Wanke, A. Wolf, and M. M. Zoeller Ohio State University, Columbus, Ohio 43210

B. Nemati, S. J. Richichi, W. R. Ross, H. Severini, and P. Skubic University of Oklahoma, Norman, Oklahoma 73019 
M. Bishai, J. Fast, J. W. Hinson, N. Menon, D. H. Miller, E. I. Shibata, I. P. J. Shipsey, and M. Yurko Purdue University, West Lafayette, Indiana 47907

S. Glenn, S. D. Johnson, Y. Kwon, ${ }^{\S}$ S. Roberts, and E. H. Thorndike University of Rochester, Rochester, New York 14627

C. P. Jessop, K. Lingel, H. Marsiske, M. L. Perl, V. Savinov, D. Ugolini, R. Wang, and X. Zhou Stanford Linear Accelerator Center, Stanford University, Stanford, California 94309

T. E. Coan, V. Fadeyev, I. Korolkov, Y. Maravin, I. Narsky, V. Shelkov, J. Staeck, R. Stroynowski, I. Volobouev, and J. Ye Southern Methodist University, Dallas, Texas 75275

M. Artuso, F. Azfar, A. Efimov, M. Goldberg, D. He, S. Kopp, G. C. Moneti, R. Mountain, S. Schuh, T. Skwarnicki, S. Stone, G. Viehhauser, and X. Xing Syracuse University, Syracuse, New York 13244

J. Bartelt, S. E. Csorna, V. Jain," K. W. McLean, and S. Marka Vanderbilt University, Nashville, Tennessee 37235

R. Godang, K. Kinoshita, I. C. Lai, P. Pomianowski, and S. Schrenk Virginia Polytechnic Institute and State University, Blacksburg, Virginia 24061

G. Bonvicini, D. Cinabro, R. Greene, L. P. Perera, and G. J. Zhou Wayne State University, Detroit, Michigan 48202

M. Chadha, S. Chan, G. Eigen, J. S. Miller, C. O’Grady, M. Schmidtler, J. Urheim, A. J. Weinstein, and F. Würthwein California Institute of Technology, Pasadena, California 91125

(CLEO Collaboration)

(Received 8 December 1997; published 24 March 1998)

\begin{abstract}
Neutrinoless 3-prong tau lepton decays into a charged lepton and either two charged particles or one neutral meson have been searched for using $4.79 \mathrm{fb}^{-1}$ of data collected with the CLEO II detector at Cornell Electron Storage Ring. This analysis represents an update of a previous study and the addition of six decay channels. In all channels the numbers of events found are compatible with background estimates and branching fraction upper limits are set for 28 different decay modes. These limits are either more stringent than those set previously or represent the first attempt to find these decays. [S0556-2821(98)04009-0]
\end{abstract}

PACS number(s): 13.35.Dx, 14.60.Fg

In the standard model of electroweak interactions the difference between the number of leptons and the number of antileptons is conserved for each generation separately. However, there is no fundamental motivation for this lepton flavor conservation in this theory because there is no symmetry associated with lepton family number. Many extensions of the standard model predict flavor violation in lepton decays. Among them are models that involve heavy neutral leptons [1-7], left-right symmetries [8-10], supersymmetry [11-14] or superstrings [15-17]. The expected decay branching fractions in these models depend on the unknown

\footnotetext{
*Permanent address: University of Texas, Austin, TX 78712.

†Permanent address: Lawrence Livermore National Laboratory, Livermore, CA 94551.

${ }^{\ddagger}$ Permanent address: BINP, RU-630090 Novosibirsk, Russia.

${ }^{\S}$ Permanent address: Yonsei University, Seoul 120-749, Korea.

"Permanent address: Brookhaven National Laboratory, Upton, NY 11973.
}

masses of proposed new particles and on the new coupling constants. The most optimistic branching fraction predictions are at the level of about $10^{-6}$. Constraints on lepton flavor violation come from studies of rare and forbidden $K, \pi$, and $\mu$ decays, $e-\mu$ conversions, neutrinoless double beta decays, neutrino oscillations, $Z \rightarrow l_{1}^{+} l_{2}^{-}$decays, and other rare processes. In particular, there are strict limits on muon neutrinoless decays: $B(\mu \rightarrow e \gamma)<4.9 \times 10^{-11}$ and $B(\mu \rightarrow e e e)<2.4$ $\times 10^{-12}$ at $90 \%$ confidence level [18]. However, lepton number violation rates may exhibit a strong dependence on mass and on generation number of the decaying particle, thus enhancing tau lepton decay rates. Also, the larger mass of the tau allows for new decay types which are kinematically forbidden for the muon.

The CLEO Collaboration has already performed comprehensive searches for neutrinoless tau decays in various channels [19-21]. The analysis presented in this paper updates the results of Ref. [19] with a more than twofold increase in the dataset size. The search also includes six additional channels. A detailed description of this analysis can be found in 
Ref. [22]. We search for tau decays into three charged particles:

$$
\begin{aligned}
& \tau^{ \pm} \rightarrow\left(l_{1} l_{2} l_{3}\right)^{ \pm}, l=e \text { or } \mu, \\
& \tau^{ \pm} \rightarrow\left(l h_{1} h_{2}\right)^{ \pm}, h=\pi^{ \pm} \text {or } K^{ \pm} .
\end{aligned}
$$

All possible combinations of final state particles and charge assignments are considered, except for those that do not conserve electric charge. Different assignments result in either lepton flavor violating decays, as in $\tau^{-} \rightarrow \mu^{-} e^{+} e^{-}$, or both lepton flavor and lepton number violating decays, as in $\tau^{-}$ $\rightarrow e^{+} \pi^{-} \pi^{-}$. We also search for $\tau$ decays into one charged lepton and one neutral meson which can subsequently decay into two charged hadrons thus resulting in three charged particles in the final state:

$$
\tau^{ \pm} \rightarrow l^{ \pm} M, M=\rho^{0}, \phi, K^{* 0}, \text { or } \bar{K}^{* 0} .
$$

The channels with two charged kaons, possibly coming from the decay of $\phi$ meson, have been searched for the first time.

The data used in this analysis were collected with the CLEO II detector [23] at the Cornell Electron Storage Ring (CESR). We use information from a 67-layer tracking system which also provides specific ionization measurements $(d E / d x)$, time-of-flight scintillation counters and a 7800crystal CsI calorimeter. These elements are inside a $1.5 \mathrm{~T}$ superconducting solenoidal magnet whose iron yoke also serves as a hadron absorber for a muon identification system. Tau leptons were produced in pairs in $e^{+} e^{-}$collisions at a center-of-mass energy of about $10.6 \mathrm{GeV}$. The data correspond to an integrated luminosity of $4.79 \mathrm{fb}^{-1}$, and the number of produced tau pairs is $4.37 \times 10^{6}$.

We follow the search method described in Ref. [19]. Signal candidate tau decays are required to produce three wellreconstructed tracks in the detector (3-prong decay). The other tau in the event must decay into a 1-prong mode, and the total visible charge must be zero. Not more than one photon candidate or background shower in the CsI calorimeter is allowed on the 3-prong side of the event.

At least one charged particle on the 3-prong side is required to satisfy electron or muon identification criteria. These criteria are more strict in the $\tau^{ \pm} \rightarrow\left(l h_{1} h_{2}\right)^{ \pm}$and the $\tau^{ \pm} \rightarrow l^{ \pm} M$ decay channels than in the $\tau^{ \pm} \rightarrow\left(l_{1} l_{2} l_{3}\right)^{ \pm}$channels because of large background from tau decays into three pions and a neutrino, in which one of the pions is misidentified as an electron or a muon. Electrons are identified by requiring that the ratio of the energy deposited by the particle in the CsI calorimeter to the momentum measured in the drift chamber is close to unity. Muon candidates are required to have a well-reconstructed track in the muon system. Charged mesons $(\pi$ or $K)$ are not positively identified, and we try all possible meson type assignments to tracks. Thus, a single event can be a candidate for more than one final state. In the channels involving neutral mesons we require the twohadron invariant mass to be consistent with that of the corresponding meson: $M_{\pi^{+} \pi^{-}}<1.2 \mathrm{GeV} / c^{2}$ for $\rho^{0}$, $0.7 \mathrm{GeV} / c^{2}<M_{\pi^{ \pm} K^{\mp}}<1.1 \mathrm{GeV} / c^{2}$ for $K^{* 0}$ and $\bar{K}^{* 0}$, $0.99 \mathrm{GeV} / c^{2}<M_{K^{ \pm} K^{\mp}}<1.05 \mathrm{GeV} / c^{2}$ for $\phi$, where the mass interval is based on the resonance width and the detector resolution.

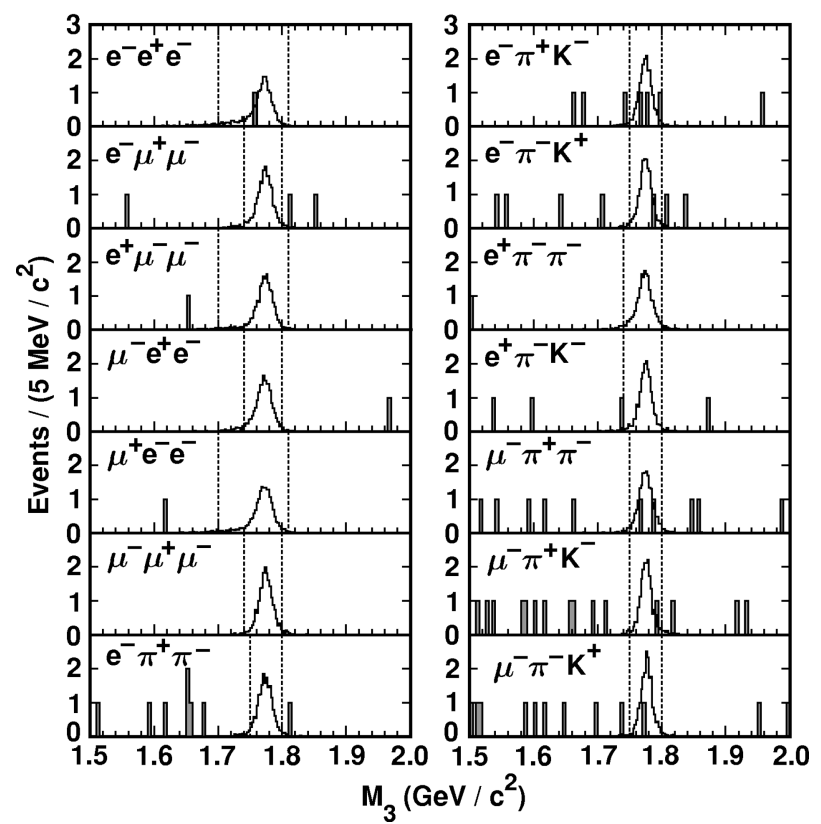

FIG. 1. Distributions of the invariant mass of the 3-prong side particles, $M_{3}$, for the data (shaded histogram) and signal Monte Carlo events (solid line). The expected signal shapes are shown with arbitrary normalization. The dotted lines indicate the boundaries of the signal regions used. See also Fig. 2.

The main backgrounds remaining after application of particle identification requirements are photon conversions in radiative Bhabha and muon pair events, two-photon processes, low multiplicity hadronic events, and $\tau \rightarrow 3 h \nu_{\tau}$ decays in which at least one hadron is misidentified as a lepton. Photon conversions in the detector material produce $e^{+} e^{-}$ pairs with small invariant masses. To suppress conversions, we consider each pair of oppositely charged particles not identified as muons under the assumption that both particles are electrons. Events are rejected if the invariant mass is less than $0.15 \mathrm{GeV} / c^{2}$ for any such pair. Two-photon processes have low values of the total transverse momentum with respect to the beam direction. In contrast, the signal events have at least one undetected neutrino on the 1-prong side which leads to transverse momentum imbalance. We reduce two-photon background contribution by requiring transverse momentum in excess of $0.2 \mathrm{GeV} / c$. Neutrino presence in the event is further exploited by requiring at least $3^{\circ}$ acollinearity between the direction of the sum of charged particles' momenta on the 3-prong side of the event and the direction of the 1-prong momentum. For neutrinoless decays the sum of the four-momenta of the particles on the 3-prong side defines the tau direction and energy. Neglecting radiative effects, the other tau in the event has an opposite momentum vector. We determine the momentum of the 1-prong charged particle in the rest system of a parent tau with boost parameters obtained by summing the four-momenta of the 3-prong side particles. Momentum values larger than half of tau mass are kinematically forbidden for tau decay products in the rest frame of decaying particle. However, presence of a neutrino on the 3-prong side of the event may result in incorrect determination of boost parameters and higher momentum. We require that the 1-prong momentum in the parent tau rest frame is less than $1 \mathrm{GeV} / c$, thus reducing background from standard tau decay modes. 


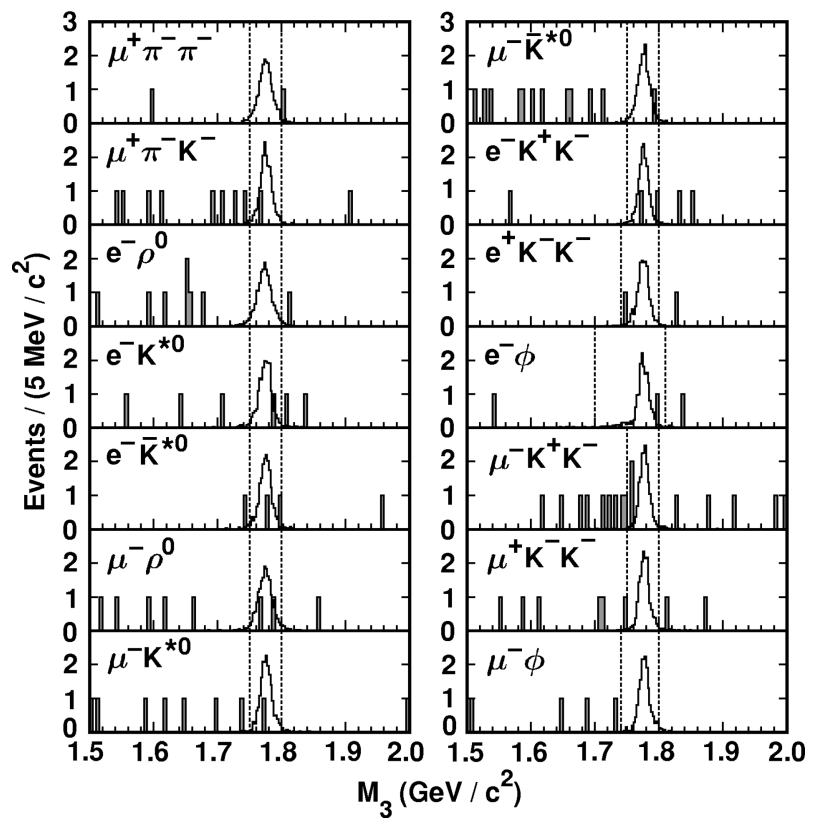

FIG. 2. Distributions of the invariant mass of the 3-prong side particles, $M_{3}$, for the data (shaded histogram) and signal Monte Carlo events (solid line). The expected signal shapes are shown with arbitrary normalization. The dotted lines indicate the boundaries of the signal regions used. See also Fig. 1.

The efficiencies of the selection criteria were estimated using 16000 Monte Carlo events for each decay channel. Phase space distributions were used to generate neutrinoless tau decays in all the channels. The KORALB-TAUOLA program package [24] was used to simulate the tau-pair production and the decay of the 1-prong tau. Subsequent meson decays and decays of the 1-prong tau were generated according to the known branching fractions [18]. Detector signals were simulated with the GEANT-based CLEO II simulation program [25].

For neutrinoless tau decays the total energy measured on the 3-prong side, $E_{3}$, must be equal to the beam energy, $E_{\text {beam }}$, and the invariant mass of the three charged particles, $M_{3}$, must be equal to the tau mass. For all channels we select rectangular signal regions in the $E_{3}-E_{\text {beam }}$ and $M_{3}$ variables taking into account detector resolution, signal efficiencies, and background levels. The signal region optimization algorithm (minimization of average expected upper limits) is described in detail in Ref. [22]. We assign the channels studied to three different groups according to their background density. For the channels with low background $\left(\tau^{-}\right.$ $\rightarrow e^{-} e^{+} e^{-}, e^{-} \phi, e^{+} \mu^{-} \mu^{-}$, and $\mu^{+} e^{-} e^{-}$, where charge conjugated modes are always implied) we define the signal region as

$$
-0.39 \mathrm{GeV}<E_{3}-E_{\text {beam }}<0.08 \mathrm{GeV}
$$

$$
1.70 \mathrm{GeV} / c^{2}<M_{3}<1.81 \mathrm{GeV} / c^{2} .
$$

For the medium background channels $\left(\tau^{-} \rightarrow e^{-} \mu^{+} \mu^{-}\right.$, $\mu^{-} \mu^{+} \mu^{-}, e^{+} \pi^{-} K^{-}, \mu^{-} e^{+} e^{-}, e^{+} \pi^{-} \pi^{-}, e^{+} K^{-} K^{-}$, and $\mu^{-} \phi$ ) we require
TABLE I. Detection efficiencies, event statistics, expected backgrounds, and upper limits for branching fractions at $90 \%$ confidence level.

\begin{tabular}{lcccc}
\hline \hline $\begin{array}{l}\text { Decay } \\
\text { channel }\end{array}$ & $\begin{array}{c}\text { Detection } \\
\text { efficiency, } \%\end{array}$ & $\begin{array}{c}\text { Events } \\
\text { observed }\end{array}$ & $\begin{array}{c}\text { Expected } \\
\text { bg events }\end{array}$ & $\begin{array}{c}\text { Upper } \\
\text { limits, } 10^{-6}\end{array}$ \\
\hline$\tau^{-} \rightarrow e^{-} e^{+} e^{-}$ & 17.0 & 1 & 0.21 & 2.9 \\
$\tau^{-} \rightarrow \mu^{-} e^{+} e^{-}$ & 16.8 & 0 & 0.18 & 1.7 \\
$\tau^{-} \rightarrow \mu^{+} e^{-} e^{-}$ & 19.5 & 0 & 0.12 & 1.5 \\
$\tau^{-} \rightarrow e^{-} \mu^{+} \mu^{-}$ & 16.5 & 0 & 0.32 & 1.8 \\
$\tau^{-} \rightarrow e^{+} \mu^{-} \mu^{-}$ & 19.9 & 0 & 0.12 & 1.5 \\
$\tau^{-} \rightarrow \mu^{-} \mu^{+} \mu^{-}$ & 15.0 & 0 & 0.11 & 1.9 \\
$\tau^{-} \rightarrow e^{-} \pi^{+} \pi^{-}$ & 13.2 & 0 & 0.43 & 2.2 \\
$\tau^{-} \rightarrow e^{-} \pi^{-} K^{+}$ & 13.0 & 1 & 0.29 & 3.8 \\
$\tau^{-} \rightarrow e^{-} \pi^{+} K^{-}$ & 13.1 & 3 & 0.42 & 6.4 \\
$\tau^{-} \rightarrow e^{-} K^{+} K^{-}$ & 11.2 & 2 & 0.29 & 6.0 \\
$\tau^{-} \rightarrow e^{+} \pi^{-} \pi^{-}$ & 15.3 & 0 & 0.22 & 1.9 \\
$\tau^{-} \rightarrow e^{+} \pi^{-} K^{-}$ & 14.0 & 0 & 0.18 & 2.1 \\
$\tau^{-} \rightarrow e^{+} K^{-} K^{-}$ & 13.0 & 1 & 0.11 & 3.8 \\
$\tau^{-} \rightarrow \mu^{-} \pi^{+} \pi^{-}$ & 8.2 & 2 & 0.57 & 8.2 \\
$\tau^{-} \rightarrow \mu^{-} \pi^{-} K^{+}$ & 6.7 & 1 & 0.48 & 7.4 \\
$\tau^{-} \rightarrow \mu^{-} \pi^{+} K^{-}$ & 6.5 & 1 & 0.49 & 7.5 \\
$\tau^{-} \rightarrow \mu^{-} K^{+} K^{-}$ & 4.5 & 2 & 0.50 & 15 \\
$\tau^{-} \rightarrow \mu^{+} \pi^{-} \pi^{-}$ & 8.6 & 0 & 0.36 & 3.4 \\
$\tau^{-} \rightarrow \mu^{+} \pi^{-} K^{-}$ & 7.0 & 1 & 0.33 & 7.0 \\
$\tau^{-} \rightarrow \mu^{+} K^{-} K^{-}$ & 4.8 & 0 & 0.35 & 6.0 \\
$\tau^{-} \rightarrow e^{-} \rho^{0}$ & 14.4 & 0 & 0.45 & 2.0 \\
$\tau^{-} \rightarrow e^{-} K^{* 0}$ & 9.5 & 1 & 0.32 & 5.1 \\
$\tau^{-} \rightarrow e^{-} \bar{K}^{* 0}$ & 9.0 & 2 & 0.32 & 7.4 \\
$\tau^{-} \rightarrow e^{-} \phi$ & 7.2 & 1 & 0.15 & 6.9 \\
$\tau^{-} \rightarrow \mu^{-} \rho^{0}$ & 10.6 & 2 & 0.43 & 6.3 \\
$\tau^{-} \rightarrow \mu^{-} K^{* 0}$ & 6.5 & 1 & 0.46 & 7.5 \\
$\tau^{-} \rightarrow \mu^{-} \bar{K}^{* 0}$ & 6.5 & 1 & 0.37 & 7.5 \\
$\tau^{-} \rightarrow \mu^{-} \phi$ & 4.1 & 0 & 0.11 & 7.0 \\
\hline \hline & & & & \\
\hline
\end{tabular}

$$
\begin{gathered}
-0.17 \mathrm{GeV}<E_{3}-E_{\text {beam }}<0.09 \mathrm{GeV}, \\
1.74 \mathrm{GeV} / c^{2}<M_{3}<1.80 \mathrm{GeV} / c^{2},
\end{gathered}
$$

and for the rest of the channels (high background group) we require

$$
\begin{gathered}
-0.09 \mathrm{GeV}<E_{3}-E_{\text {beam }}<0.06 \mathrm{GeV}, \\
1.75 \mathrm{GeV} / c^{2}<M_{3}<1.80 \mathrm{GeV} / c^{2} .
\end{gathered}
$$

The 3-prong invariant mass distributions of events satisfying all background suppression criteria and lying within the $E_{3}-E_{\text {beam }}$ limits defined above are shown in Figs. 1 and 2 , together with the expected signal shapes generated by the Monte Carlo simulation. There are 14 events in the data which satisfy all the selection criteria, including the 3-prong invariant mass requirement, in at least one channel. Seven of these events satisfy the selection criteria in two different channels (notably, most candidates for $\tau^{ \pm} \rightarrow l^{ \pm} M$ decays also qualify for corresponding non-resonant decays), and 1 event satisfies the selection criteria in three different channels. In each channel the number of data events inside the signal region is consistent with the estimated background 
level. The largest deviation is observed in the $\tau^{-}$ $\rightarrow e^{-} \pi^{+} K^{-}$channel which has three events while the expected background is 0.42 events. The probability of such a deviation or larger is about $1 \%$, if calculated according to Poisson statistics. However, with 28 channels investigated, such fluctuations can be expected in one or two of them. In addition, Poisson statistics may fail to provide an accurate consistency check because, due to the small size of the event sample remaining after background suppression, we use the same sideband data for both signal region optimization and background estimation.

In each channel we calculate the branching fraction upper limit at the $90 \%$ confidence level according to the convention adopted by the Particle Data Group [18], and we do not attempt to subtract the background. Systematic errors in this analysis arise from uncertainties in our knowledge of the luminosity, track reconstruction efficiency, lepton identification efficiency, and 3-prong energy and invariant mass resolutions. Combined together, they are conservatively estimated to increase branching fraction upper limits by $10 \%$. We do not assign any systematic error due to model dependence. However, we emphasize that our limits depend on the assumed angular and momentum distributions of the decay particles.

The final results are summarized in Table I, together with the detection efficiencies obtained for each mode from
Monte Carlo simulations and with the numbers of events observed in the data. The limits obtained in this analysis are more stringent than those obtained previously [18]. In addition, the limits on $B\left(\tau^{-} \rightarrow \mu^{+} e^{-} e^{-}\right)$and $B\left(\tau^{-}\right.$ $\rightarrow e^{+} \mu^{-} \mu^{-}$) are the most stringent limits to date on lepton number violation in $\tau$ decays. In SUSY with broken $R$-parity $[13,14]$ and in the model with radiatively generated lepton masses from Ref. [2] the obtained results provide constraints on model parameters. In models with heavy neutral leptons [5-7] the experimental limits are close to the allowed range of neutrinoless $\tau$ decay rates.

We gratefully acknowledge the effort of the CESR staff in providing us with excellent luminosity and running conditions. J.P.A., J.R.P., and I.P.J.S. thank the NYI program of the NSF, M.S. thanks the PFF program of the NSF, G.E. thanks the Heisenberg Foundation, K.K.G., M.S., H.N.N., T.S., and H.Y. thank the OJI program of DOE, J.R.P., K.H., M.S. and V.S. thank the A. P. Sloan Foundation, R.W. thanks the Alexander von Humboldt Stiftung, M.S. thanks Research Corporation, and S.D. thanks the Swiss National Science Foundation for support. This work was supported by the National Science Foundation, the U.S. Department of Energy, and the Natural Sciences and Engineering Research Council of Canada.
[1] M. C. Gonzalez-Garsia and J. W. F. Valle, Mod. Phys. Lett. A 7, 477 (1992).

[2] G.-G. Wong and W.-S. Hou, Phys. Rev. D 50, 2962 (1994).

[3] A. Pilaftsis, Mod. Phys. Lett. A 9, 3595 (1994).

[4] J. Hisano et al., Phys. Lett. B 357, 579 (1995).

[5] A. Ilakovac and A. Pilaftsis, Nucl. Phys. B437, 491 (1995).

[6] A. Ilakovac, B. A. Kniehl, and A. Pilaftsis, Phys. Rev. D 52, 3993 (1995).

[7] A. Ilakovac, Phys. Rev. D 54, 5653 (1996).

[8] R. N. Mohapatra, Phys. Rev. D 46, 2990 (1992).

[9] R. N. Mohapatra, S. Nussinov, and X. Zhang, Phys. Rev. D 49, 2410 (1994).

[10] S. Pastor, S. D. Rindani, and J. W. F. Valle, FTUV-97-23, hep-ph/9705394 (1997).

[11] J. C. Romao, N. Rius, and J. W. F. Valle, Nucl. Phys. B363, 369 (1991).

[12] G. Bhattacharyya and D. Choudhury, Mod. Phys. Lett. A 10, 1699 (1995).

[13] D. Choudhury and P. Roy, Phys. Lett. B 378, 153 (1996).
[14] J. E. Kim, P. Ko, and D.-G. Lee, Phys. Rev. D 56, 100 (1997).

[15] S. Kelley et al., Nucl. Phys. B358, 27 (1991).

[16] R. Arnowitt and P. Nath, Phys. Rev. Lett. 66, 2708 (1991).

[17] J. Wu, S. Urano, and R. Arnowitt, Phys. Rev. D 47, 4006 (1993).

[18] Particle Data Group, R. M. Barnett et al., Phys. Rev. D 54, 1 (1996).

[19] J. Bartelt et al., Phys. Rev. Lett. 73, 1890 (1994).

[20] K. Edwards et al., Phys. Rev. D 55, 3919 (1997).

[21] G. Bonvicini et al., Phys. Rev. Lett. 79, 1221 (1997).

[22] I. Volobouev, Ph.D. thesis, Southern Methodist University, SMUPHT/97-2 (1997).

[23] Y. Kubota et al., Nucl. Instrum. Methods Phys. Res. A 320, 66 (1992).

[24] S. Jadach and Z. Wạs, Comput. Phys. Commun. 64, 267 (1991); S. Jadach et al., ibid. 76, 361 (1993).

[25] The CLEO II detector simulation is based on the GEANT software package: R. Brun et al., GEANT version 3.15, CERN DD/ EE/84-1. 